\title{
Software Sub-system in Loading Automatic Test System for the Measurement of Power Line Filters
}

\author{
Bo $\mathrm{Yu}^{1 *}$, Lin $\mathrm{Fu}^{1}$ and Adam Barker ${ }^{2}$ \\ ${ }^{1}$ Chengdu Technology University Chengdu, 611730, China \\ ${ }^{2}$ School of Computer Science, st andrews, Uk \\ Corresponding Email: 14392926@qq.com
}

\begin{abstract}
The loading automatic test system for measurement of power line filters are in urgent demand. So the software sub-system of the whole test system was proposed. Methods: structured the test system based on the virtual instrument framework, which consisted of lower and up computer and adopted the top down approach of design to perform the system and its modules, according to the measurement principle of the test system. Results: The software sub-system including human machine interface, data analysis and process software, expert system, communication software, control software in lower computer, etc. had been designed. Furthermore, it had been integrated into the entire test system. Conclusion: This sub-system provided a fiendly software platform for the whole test system, and had many advantages such as strong functions, high performances, low prices. It not only raises the test efficiency of EMI filters, but also renders some creativities.
\end{abstract}

Keywords. Loading, automatic test system, software sub-system, power line filter

\section{Introduction}

Power line filter plays an important role in each electrical and electric system for suppressing the EMI(Electromagnetic Interference) in order to meet the requirements of EMC(ElectroMagnetic Compatibility) Certification standards. The more strict rules of the standards are, the more extensive those filters are used, and the higher quantities of the filters have been demanded. How to make an efficient and accuracy test for the filters becomes an urgent job, which must not only design reliable measurement process but also implement the whole integrated test system to ensure the conformity of the electrical technical parameters and performance indexes of the filters. Those technical specifications include rated voltage/current, working voltage/current, withstand voltage, residual voltage, leakage current, insertion loss (IL), etc. Each specification has been ruled by certification standard(s) for its value or measuring method. GB7343-1987[1] rules the methods of measurement of suppression characteristics of passive radio interference filters and suppression components over the frequency range of $10 \mathrm{khz}$ to $30 \mathrm{Mhz}$, and MIL-STD-220B[2] stipulates the methods for IL testing when filter is under full loading, while CISPR 17[3-4] fixes the approximate worst case impedance IL testing beyond the standard test method which uses a calibrated $50 \Omega$ signal source and a $50 \Omega$ receiver. The main problem is, faced with those specifications which use different test methods and different kinds of equipments, the whole test process had been separated to some individual measurement, and before now, each test of the index or indicator of the filters had been performed manually, in which there always some defaults been made, even catastrophic effect such as electric shock for operation staff, or physical damage for the equipments or devices under test. Thus there has so far seldom find the papers reporting and discussing automatically test for specifications of the filters except some researches for IL measurement only in which absolutely do not refer as loading test, not to mention that it integrate loading test to implement automatic measurement.

This paper addresses the software design in an actual automatic test system for main specifications stated above especially the loading IL automatically testing for the filter. The aims of software design in the test system are not only to overcome the shortcomings of traditional manually testing but also provide some advanced features and performances for the whole system and the test program. First, it sets up a friendly software platform to implement whole automatic specification test for each filter when it has been installed into the homemade test box which plays an important role in the testing configuration. Second, it performs an automatically test procedure according to the turns of index for DUT. Third, it realizes some intelligent function such as provides some redesign propositions for those unqualified DUT.

\section{Framework and principle of the whole system}




\subsection{Framework}

The whole test system was composed of an upper and lower computer architecture which based on the virtual instrument framework as shown in figure 1. Under the framework the hardware sub-system consisted of partly various commercial off the shelf(COTS) devices and equipments including PC, impedance tester, test voltage tester, etc., and partly homemade modules in which the key feature was that it integrated severally the signal generator and the receiver on a single card based on PCI bus plug in the PC to replace the vector network analyzer used in traditional manually test method, reduced the cost of the test system, and made the test more flexible accompanied with its more functions and no less performances. Note, the links among COTS equipments and homemade modules are totally standard interfaces, junctors, and shield cables.

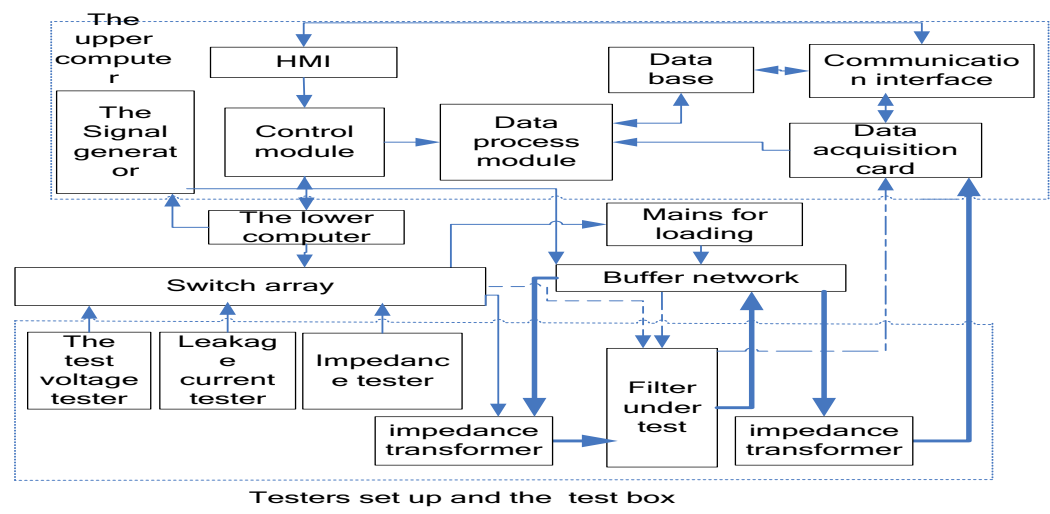

Fig. 1. Framework of the test system

\subsection{Principle}

As stated above, we must test multiple index for each filter, and the testing principles and equipments or instruments are different, so the test modes or methods are different respectively. Take IL test for instance, the principle of the system is: the operation command Issued by the upper computer, and the lower computer send the test signals which output by the signal generator to the filter under test in the test box via switch array, then the receiver collect the signals output by filter and convey them to the PC via communication interface. When the impedance between source and input port of filter and output vs. load are matching, signal follows the dotted line while the approximate worst case it follows the heavy line in figure 1 .

As we know, the IL of EMI filter separated to two kinds, one is the common-mode (CM), and the other is the differential-mode (DM). Therefore the IL test configuration of hardware for EMI filters measurement must accordingly set up two forms. The CM set up as figure 2, while DM set up as figure 3. The flow chart of IL test shown as figure 4.

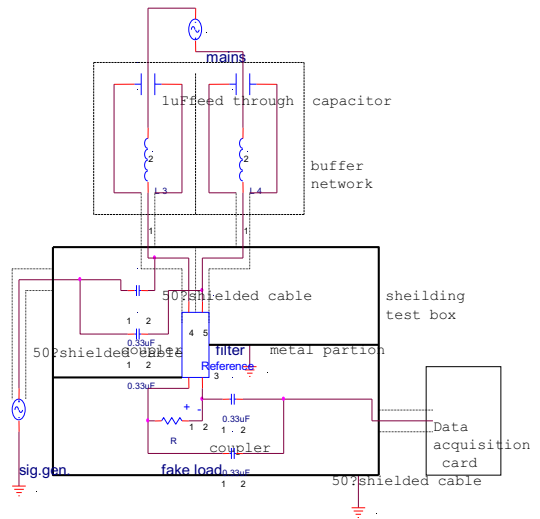

Fig.2. Hardware set up for IL of CM testing

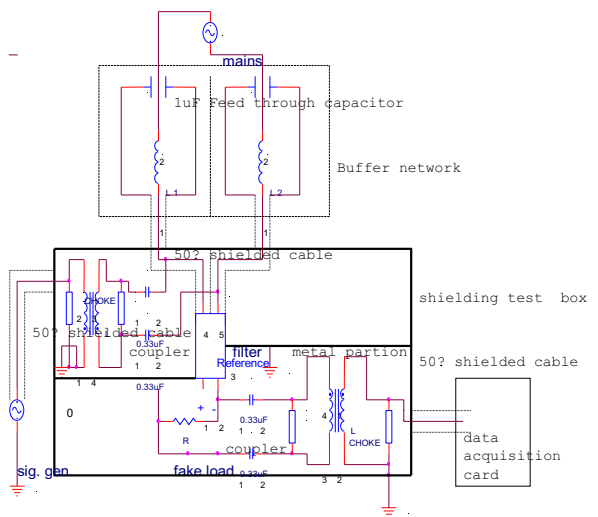

Fig.3. Hardware set up IL of DM testing

in which the automatic test process of IL is illustrated eventually, and the other index procedures such as insert loss, and test voltage, as well as leakage current are also including in the flowchart. 


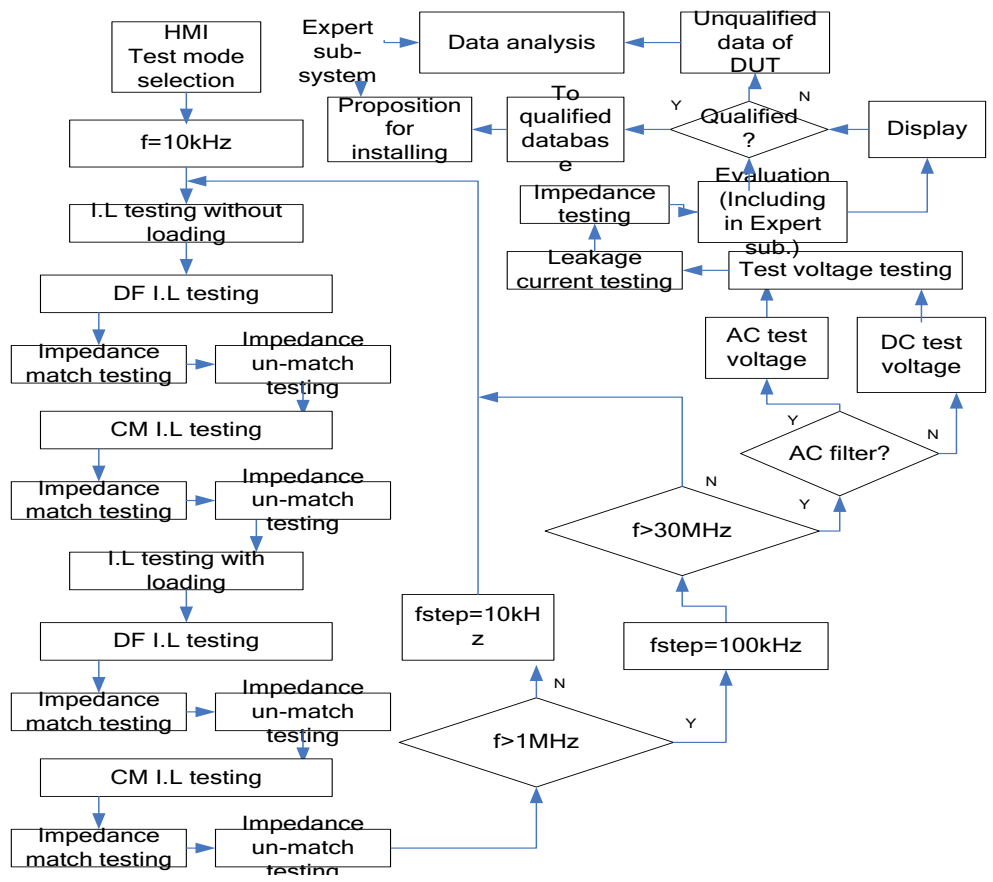

Fig. 4. Main index testing flowchart of the software

From figure 4, we note that there is an expert sub-system in the software, which is the one of our innovation points for the test system. Based on the expert sub-system, the test system can evaluate the quality of each DUT automatically, and further, it can diagnose the causes leading to invalidation for each unqualified DUT according to analysis module in the software sub-system.

\section{Main intelligent modules of the software sub-system}

The data sent from acquisition card which based on PCI bus plug in must have been analyzed using the intelligent modules integrated in the software. So the intelligent module plays an important role in the whole system, in which there consist with three parts, one is the I.L spectrum analysis module, second is the evaluation module, and third is the proposition module.

\subsection{I.L spectrum analysis}

The data analysis and process is the key component of the system, in which there are many methods including traditional time domain approaches and frequency domain approaches, and modern methods used to analyze and process the data from different input including output of data acquisition card, database, and internet. For the output of data acquisition card, there is a real time event, and for the data from database, there is an analysis and process for the third part data or the historical data. For the data from the internet, there is not only a remote measurement but also a real time event. According to the results of analysis module, the software can display time domain waves, spectrum plot.

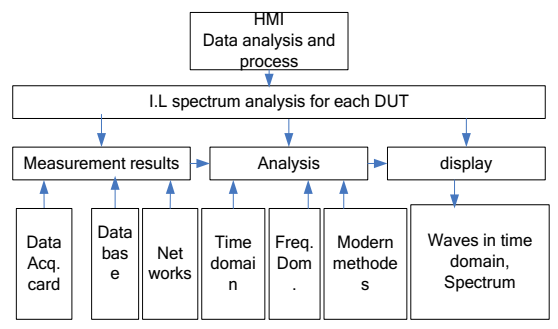

Fig.5. I.L analysis module

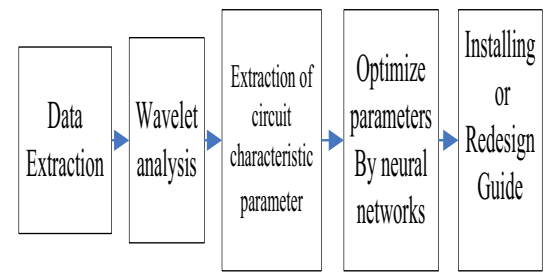

Fig. 6. Diagnosis module

\subsection{Evaluation module}


The evaluation module intellectually compares the data between test and analysis results and the database which keeps the records of good and standardized parameters for the DUT, furthermore, it makes a judgment that it is belong to unqualified or qualified ones for each DUT automatically. Finally, it stores the data into different database accordingly, noting that there is an intelligent discriminating between better DUT and good DUT in critical area, the data of later must send to the diagnosis module to obtain the installing guide In order to facilitate the efficiency of the DUT in the real system.

\subsection{Diagnosis module}

The diagnosis module shown as figure 6, it employs wavelet analysis and neural networks to analyze the DUTs which been judged to unqualified or in the critical area of good ones.

\subsection{Software modules in the lower Computer}

Software modules in the lower computer including MCU main module, up computer instruction handout module, switch array control module, etc. the MCU main module is the kernel of lower computer software, which operating the whole lower computer. The module of up computer instruction handout gives away the instructions from up c0puter through MCU main module to the modules implementing its function respectively. And, as stated above, to achieve all testing procedures, the whole system must employ some other instruments or equipments, so there must adopt multiple switches to change the test functions. Further, in order to deals with the approximate worst-case test method for testing the insertion loss of the EMI filters, the $0.1 \Omega$ and $100 \Omega$ and vice versa impedances usually used for this testing. Thus it must design the impedance transformer network which needs switches in proper order on or off according to the test process. The software of switch array control module is designed accordingly.

\section{Results and conclusion}

\subsection{Results}

The whole sub-system was designed perfectively, according to different testing function, the software had some different HMI. Noting that the software was designed for Chinese, so the language in the software is Chinese.

As shown in figure 7, there is a filter just under test been testing it I.L parameters. And as shown in figure 8, there is the data check HMI which indicated the output of testing.

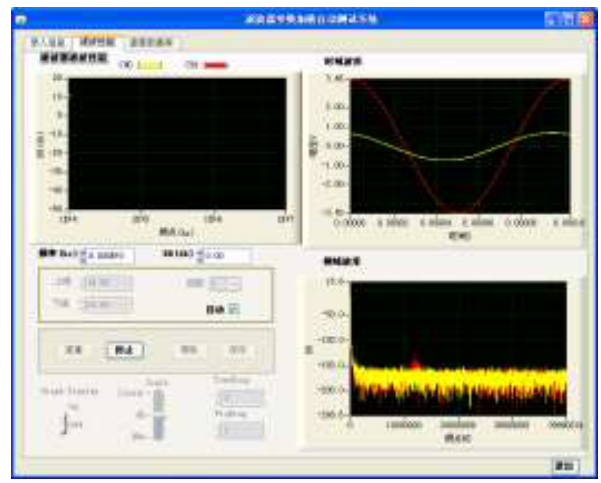

Fig.7. The HMI of I.L testing

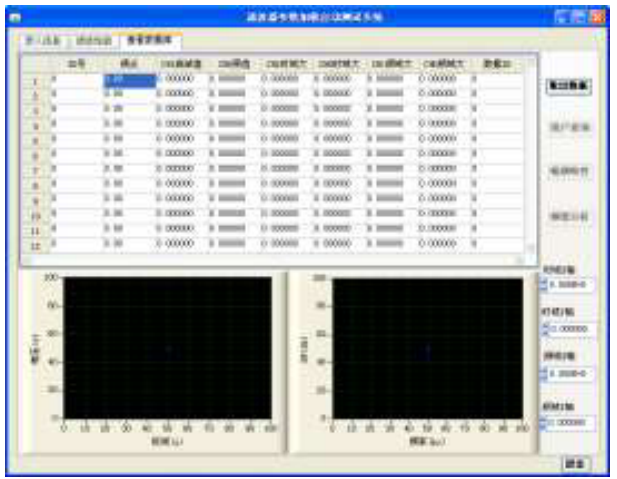

Fig.8. The data check function HMI

\subsection{Conclusion}

The software sub-system rendering a friendly and efficient platform to implement the automatic loading test for power line filters. Its main creativity is that it integrated the loading test and automatic test synchronously with its virtual instrument framework, and based on the framework, the strong power of the software in the test system can perform more intelligent. This sub-system had been granted with the utility model patent certificate for the whole system, which patent number is ZL 201420203665.8. 


\section{References}

1. GB 7343-87, methods of measurement of suppression characteristics of passive radio interference filters and suppression components over the frequency range of $10 \mathrm{khz}$ to $30 \mathrm{Mhz}$.

2. MIL-STD-220B, METHOD OF INSERTION LOSS MEASUREMENT

3. IEC CISPR 17, Methods of measurement of the suppression characteristics of passive radio interference filters and suppression components (1981)

4. Jiri DRINOVSKY, Zdenek KEJIK, etc. Insertion Loss Estimation of EMI Filters in Unmatched Input Output Impedance System. RADIOENGINEERING, 20, 1, APRIL (2011):295-298

5. Zhang Shuping, Zhao Yajun. Analysis of manual test mode for EMI filter of insert loss. Inner Mongolia Science Technology \& Economy, No.16 (2008): 114-115

6. Ma Jianing, Song Bo. Automatic test system for insertion loss in EMI power filter. Intellectual Property Office of the people's Republic of China. CN203191451U. 2013.09

7. Ma Jianing, Song Bo. Automatic test system and methods for insertion loss in EMI power filter. Intellectual Property Office of the people's Republic of China.CN102944752A. 2013.02

8. Liu Bin, Li Gang, Jiang Quanxing. Design of Insertion Loss Testing System of EMI Power line Filter. Safty \&EMC. No.2 (2005).

9. Wang Meng, Zhou Ping. Design of Automatic Test System for Insertion Loss in EMI Power Filter. Electronic quality, No.09 (2008):71-76

10. Ye Fen. A automatic method for EMI filter testing. Intellectual Property Office of the people's Republic of China.CN1657964A. 2005.05

11. Cao Quanming, Cai Dongxian. A automatic testing system for EMI filter. Intellectual Property Office of the people's Republic of China. CN101315404B. 2010.07.

12. LiuHao, Wei Lili, Zhong Xiaoyan. EMI power line filter automatic testing system based on virtual instrumentation. measurement techniques, No.2 (2008):10-13

13. Liu Hao, Ding Xue. Research of insert loss testing techniques for EMI power line filter. Electronic testing. No.7, (2007):51-53

14. Qiu Yang. A testing equipment of current loading for power line filters. Intellectual Property Office of the people's Republic of China.CN201607516U. 2010.10.

15. Zhang Jinkui. Development and Analysis of the test equipment of EMI filter with current applied. Master Thesis of Southeast University (2007).

16. Liu Bin. DESIGN OF LOAD TESTING SYSTEM OF EMI POWER LINE filter. Master Thesis of Southeast University (2007)

17. Liu Song. The integrating machine of filter reshaping automatic testing. Intellectual Property Office of the people's Republic of China. CN201985231U .2011.09

18. Qiu Yang. The design method and multiple function equipment of power line filter electrical performance. Intellectual Property Office of the people's Republic of China.CN101788631B. 2014.1

19. Tang Shiping,Yang Jingfa, Huang Jian. Research of testing method for insret loss feature of EMI filte. Saftye \&EMC, No.2 (1999): 26-37 